\title{
Cities as Evolutionary Systems in Random Media*
}

\author{
Leonid Bogachev \\ Department of Statistics, University of Leeds, Leeds LS2 9JT, UK. \\ E-mail: bogachev@maths.leeds.ac.uk
}

\begin{abstract}
The purpose of the paper is to discuss some potential applications of random media theory to urban modelling, with the emphasis on the intermittency phenomenon. The moment test of intermittency is explained using the model of continuous-time branching random walk on the integer lattice $\mathbb{Z}^{d}$ with random branching rates. Statistical moments of the population density are studied using a Cauchy problem for the Anderson operator with random potential. The Feynman-Kac representation of the solution is discussed, and Lyapunov exponents responsible for the super-exponential growth of the moments are evaluated. The higher-order Lyapunov exponents are also obtained. The results suggest that the higher-order intermittency is reduced, in a sense, to that of the mean population density.

Key words and phrases: Urban modelling, theory of random media, intermittency, branching random walk, quenched moments, annealed moments, Lyapunov exponents.
\end{abstract}

\section{Introduction}

There has been a long tradition in urban and regional science to approach the mathematical modelling of urban processes in the spirit of classical mathematical physics, by developing various deterministic (sometimes, quite sophisticated) models, aiming to capture the multi-component, dynamically variable nature of cities by augmenting the equations with suitable feedback mechanisms, akin to reaction-diffusion models or predator-prey models (see, e.g., Wilson 1981; Bracken \& Tuckwell 1992, and further references therein). Although often useful in practical terms, the efficiency of such models proved to be limited to short spatio-temporal scales, hence there was the growing understanding among both theorists and practitioners that the deterministic approach was conceptually flawed, as it was missing some intrinsic, significant features of cities as agglomerates with formidably complex structure and intricate dynamics. Gradually, the simplistic paradigm of deterministic modelling has been superseded by the complexity paradigm, and in the last three decades or so it has become conventional to think of modern cities and regions as complex spatial systems (see Wilson 2000; Portugali 2000). More recently, ideas from the theory of self-organization proved to be useful in attempts to explain a familiar phenomenological picture of the city as an apparently "self-organizing" system, proceeding from agent-based models (see Portugali 2000).

\footnotetext{
*Research supported in part by DFG Grant 436 RUS 113/722.
} 
One significant feature characteristic to all such systems is the inherent uncertainty of their spatio-temporal dynamics, driven by various transport processes arising through interaction between "agents" at different structural levels and scales. In the urban context, this is exemplified by the competition between agents (individuals or organizations) via exchange of resources (e.g., land, labour, finances, goods, and information), which involves spatial mobility (transport networks and traffic, commuting flows, etc.) and population dynamics (demography, migration, etc.).

It is important to recognize that cities as systems are always non-closed due to interaction with the external medium ("environment"), which in turn may be random or fluctuating in its own right. This suggests that any conceptual urban model should involve appropriate random processes evolving in a random medium. One example of such processes is provided by random walks in random environments, where the usual random walk as a model of spatial motion of agents is modified so that the probabilities of jumps (or, in a continuous-time setting, the jumping rates) depend in a random fashion on the agent's current location (Bogachev 2006). In situations where, in addition, there is a possibility of local reproduction or annihilation of agents (which may be modelled by branching random walk), it may be necessary to allow the branching probabilities (rates) to be space-dependent and random.

Modern theory of random media provides an adequate framework to model such systems (see, e.g., Zeldovich et al. 1988; Molchanov 1991). Typically, evolutionary processes in random media are driven by the two competing mechanisms: (1) selfaveraging (via diffusion, dissipation, etc.) and (2) self-organization (via interaction, branching, local reactions, etc.). In the short term, when fluctuations of the medium are moderate enough, self-averaging (homogenization) usually prevails, allowing one to replace the complicated random medium by a much simpler effective non-random medium (see Kozlov 1985; Molchanov 1991). Loosely speaking, such a situation is analogous to the asymptotic normality in probability theory, where the combination of lots of small random factors generates a Gaussian distribution of fluctuations around non-random "mean". In fact, the idea of homogenization underlies the classical mathematical physics and dates back to the pioneering work of Boltzmann (thermodynamics), Maxwell (electromagnetic dynamics) and Rayleigh (heat propagation); see a historical review and an account of modern development in Molchanov (1991).

However, over the past three decades or so, it has been observed that in many cases, due to statistically rare but extremely large fluctuations of the random environment, the interplay between self-averaging and self-organization may lead to anomalous effects that cannot be predicted or explained via the averaged approach, such as localization, intermittency, non-classical diffusion, etc. (see Zeldovich et al. 1988; Bouchaud \& Georges 1990; Molchanov 1991, 1994, and further vast bibliography therein).

In spatio-temporal modelling of cities as complex systems, of particular interest is the intermittency phenomenon. This term, originally coined in the theory of hydrodynamic turbulence (see Monin \& Yaglom 1975) and later on made popular in synergetics and nonlinear science (see Haken 1978; Mikhailov \& Loskutov 1991), refers to the development of highly irregular structures in space and time, featured by a hierarchy of extremely high and sparse peaks ("spikes") on a relatively low-profile background. Intermittency is typical for complex processes in many areas including chemical physics 
(strong centers in catalytic reactions), biology (formation of living organisms), ecology (propagation of species in the biosphere), genetics (gene expression on DNA microarrays), hydrodynamics (hierarchy of vortices in Kolmogorov-Obukhov's theory of hydrodynamic turbulence), astrophysics (formation of galaxies, stars and planets), and economics (accumulation and redistribution of wealth and resources; modern globalization), to mention but a few (see Zeldovich et al. 1988; Bouchaud \& Georges 1990; Molchanov 1991, and further references therein).

In the urban context, an example of intermittency is readily provided by cities as they stand, representing high, relatively localized peaks of the population density (cf. the hierarchy "villages - towns - cities - megacities - megalopolises"). Let us point out that first attempts to allow for stochastic factors in urban and regional models were made by collaborators within the Brussels school (see Allen et al. 1978; Allen \& Sanglier 1979) who applied Prigogine's ideas (see Nicolis \& Prigogine 1977, 1989) to urban systems. A critical survey and discussion of the Brussels school's approach can be found in Wilson (1981). In these works it was observed, mainly via numerical integration and computer simulations, that stochastic dynamics generates hierarchically-structured spatial patterns, but the role of randomness has largely remained obscure. Among more recent approaches, let us mention the thought-provoking work by Zanette \& Manrubia (1997, 1998) and Manrubia \& Zanette (1998) on application of intermittency ideas in urban modelling in an attempt to explain the Zipf law of the Pareto-type distribution of the size of large cities. An interesting example of intermittency has been recently reported by Giardina \& Bouchaud (2003) for an agent-based market model.

The main purpose of the present paper is to draw a wider attention of urban and regional scientists to possible anomalies caused by the indispensable randomness of the environment. In particular, we aim to explain the powerful yet simple moment test of intermittency, proposed by Zeldovich et al. (1988) and developed further by Gärtner \& Molchanov (1990) and Molchanov (1991, 1994). Heuristically, the qualitative picture of intermittency suggests that, due to high peaks, intermittent spatial structures are characterized by an anomalous ratio between consecutive statistical moments of the evolving field (so that, e.g., the second-order moment grows much faster than the square of the first-order one, etc.). We shall illustrate this picture using a simple model of branching random walk with random birth-and-death rates.

The paper is organized as follows. In Section 2 we describe our basic model of continuous-time branching random walk in $d$ dimensions. In Section 3, we consider the mean population size $m_{1}$ in a fixed (quenched) branching medium and derive Kolmogorov's equation for $m_{1}$ as a function of time and the initial position of the ancestor. The Feynman-Kac representation of the solution, which plays the crucial role in our analysis, is explained in Section 4. In Section 5 the long-term behaviour of $m_{1}$ is discussed, using the so-called annealed moments $\left\langle m_{1}^{p}\right\rangle$ obtained by further averaging over the medium. In particular, the Lyapunov exponents are computed explicitly in the Weibull case. It follows that the moments $\left\langle m_{1}^{p}\right\rangle$ grow progressively, and in Section 6 we explain why this is an evidence of strong intermittency of $m_{1}$. In Section 7 , these results are extended to higher-order moments of the population size, which prove to be intermittent as well. In Section 8 we note a remarkable identity between the Lyapunov exponents of various orders, which suggests that the higher-order intermittency is in 
a sense reduced to the first-order intermittency. Finally, concluding remarks are made in Section 9.

\section{The model}

In a simplified setting, we may think of an evolutionary spatial system (e.g., a city) as being driven by certain "agents" which move randomly in space and may interact locally with the environment (but not with each other). To be more specific, we suppose that the spatial motion is of type of diffusion, which will be modelled via continuous-time simple symmetric random walk on the integer lattice $\mathbb{Z}^{d}(d \geq 1)$ with jumping rate $\kappa>0$. The latter means that during a small amount of time $h \rightarrow 0$, the walk currently at site $x \in \mathbb{Z}^{d}$ can jump to an adjacent site $x^{\prime}\left(\left|x^{\prime}-x\right|=1\right)$ with probability $(2 d)^{-1} \kappa h+o(h)$ (see Fig. 1). The remaining option, of course, is to stay at the current location over time $h$, which has probability $1-\kappa h+o(h)$. (Here and below, the notation $y=o(h)$ means that $y=y(h)$ is much smaller than $h$, that is, $y(h) / h \rightarrow 0$ as $h \rightarrow 0$.) As is well known from the standard theory of Markov chains (see, e.g., Gihman \& Skorohod 1975), an equivalent description of the random walk's local dynamics is that it spends at each site an exponentially distributed random time (with mean $1 / \kappa$ ), thereafter jumping to one of the $2 d$ adjacent sites chosen at random, with equal probabilities $(2 d)^{-1}$.
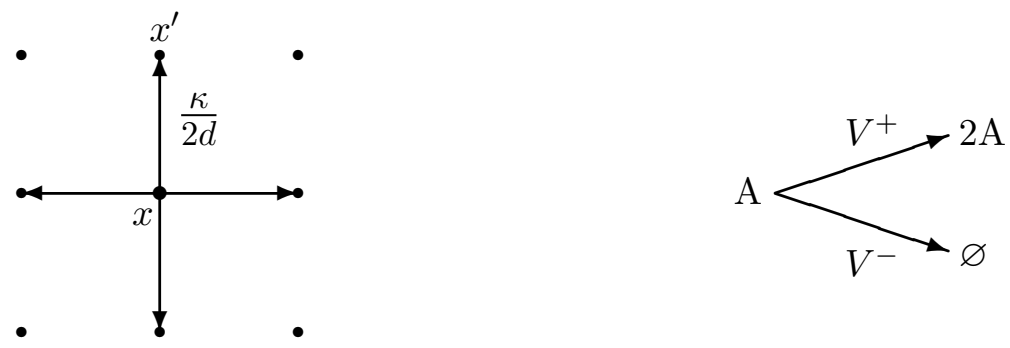

Fig. 1. Random walk transitions $(d=2)$

Fig. 2. Branching transitions

Furthermore, we suppose that the local variability of the population is governed by a birth-and-death process, whereby an agent may be either replaced by two new agents (at reproduction rate $V^{+}$) or removed from the system (at annihilation rate $V^{-}$). Possible branching transitions of an agent at each locus are schematically shown in Fig. 2. It is assumed that the branching rates are variable in space: $V^{ \pm}=V^{ \pm}(x)$ $\left(x \in \mathbb{Z}^{d}\right)$, and random (hence random branching medium). The probability distribution of the branching medium will be denoted by $\mathbb{P}$, and expectation with respect to $\mathbb{P}$ by the angular brackets, $\langle\cdot\rangle$.

Thus, given a realization $\omega$ of the random medium (i.e., a collection of the branching rates $\left.V^{ \pm}(\cdot)\right)$, an agent, currently at site $x \in \mathbb{Z}^{d}$, during a small time $h \rightarrow 0$ is replaced by two descendants with probability $V^{+}(x) h+o(h)$, dies with probability $V^{-}(x) h+o(h)$, or else stays intact, with probability $1-V^{+}(x) h-V^{-}(x) h+o(h)$. Similarly to the random walk's description above, this implies that the random time until branching transformation has exponential distribution with parameter $V^{-}(x)+V^{+}(x)$, and when 
it expires the agent either splits into two or dies, with probabilities proportional to $V^{+}(x)$ or $V^{-}(x)$, respectively.

As usual, it is assumed that the mechanisms of random walk and branching act independently of each other, which amounts to saying that exponential random "clocks" measuring time until jumping and branching are statistically independent. Therefore, the dynamics of the population ensemble proceeds on the "microscopic" time scale according to the following rule. If at time $t$ an agent is located at site $x$, then during a small subsequent amount of time $h>0$, with probability $(2 d)^{-1} \kappa h+o(h)$ it can jump to an adjacent site $x^{\prime}\left(\left|x^{\prime}-x\right|=1\right)$, with probability $V^{+}(x) h+o(h)$ it is replaced by two agents at the point $x$, or otherwise, with probability $V^{-}(x) h+o(h)$ the agent dies. Accordingly, with probability $1-\kappa h-\left(V^{+}(x)+V^{-}(x)\right) h+o(h)$ the agent experiences no transformations during the whole time $h$. The following table contains the list of all "elementary" transitions (up to probabilities of order of $h \rightarrow 0$ ):

Table 1

\begin{tabular}{|l|c|}
\hline \multicolumn{1}{|c|}{ Transition } & Probability \\
\hline Jump to adjacent site & $\frac{\kappa}{2 d} h+o(h)$ \\
Split into two & $V^{+}(x) h+o(h)$ \\
Die & $V^{-}(x) h+o(h)$ \\
Stay intact & $1-\left(\frac{\kappa}{2 d}+V^{+}(x)+V^{-}(x)\right) h+o(h)$ \\
\hline
\end{tabular}

Newly born agents evolve by the same rule, independently of the other agents and the past history. We also impose the initial condition by assuming that at time $t=0$ there is a single agent in the system located at some point $x \in \mathbb{Z}^{d}$.

In what follows, we denote by $\mathrm{P}_{x}^{\omega}$ the quenched probability law of this process, and by $\mathrm{E}_{x}^{\omega}$ the corresponding expectation. (The label $\omega$ refers to a fixed realization of the branching medium $V^{ \pm}(\cdot)$, whereas the subscript $x$ indicates the initial position of the single original agent.)

\section{First-order quenched moment}

Let us denote by $X_{t}(y)$ the number of agents at time $t \geq 0$ at site $y \in \mathbb{Z}^{d}$, and consider also the total population size $X_{t}:=\sum_{y} X_{t}(y)$. Since at time $t=0$ there is one agent in the system placed at point $x \in \mathbb{Z}^{d}$, we note that

$$
X_{0}=1, \quad X_{0}(y)=\delta_{x}(y),
$$

where $\delta_{x}(y)$ is the Kronecker symbol taking values 1 and 0 according as $x=y$ or $x \neq y$, respectively. 
Fixing a realization $\omega$ of the branching medium, let us consider the quenched statistical moments of the first order:

$$
m_{1}(t, x, y):=\mathrm{E}_{x}^{\omega} X_{t}(y), \quad m_{1}(t, x):=\mathrm{E}_{x}^{\omega} X_{t} .
$$

Backward Kolmogorov's equations for these functions can be derived in the usual way, by decomposing with respect to possible transitions during the initial time $h \rightarrow 0$. For example, for the mean population size $m_{1}(t, x)$ we can write, using Table 1:

$$
\begin{aligned}
m_{1}(t+h, x)= & \frac{\kappa h}{2 d} \sum_{\left|x^{\prime}-x\right|=1} m_{1}\left(t, x^{\prime}\right)+2 m_{1}(t, x) V^{+}(x) h \\
& +\left[1-\kappa h-V^{+}(x) h-V^{-}(x) h\right] m_{1}(t, x)+o(h) .
\end{aligned}
$$

Indeed, the sum over the nearest neighbours $x^{\prime}$ in (2) accounts for possible jumps from $x$ to $x^{\prime}$, which amounts to re-starting the process at point $x^{\prime}$, while the second term in (2) represents the possibility of branching, so that at time $h$ we have two agents at $x$ and hence the total mean population size after subsequent time $t$ will be double the $m_{1}(t, x)$. Subtracting $m_{1}(t, x)$ from both parts of (2), dividing by $h$ and passing to the limit as $h \rightarrow 0$, we arrive at the equation

$$
\left\{\begin{array}{l}
\partial_{t} m_{1}=\kappa \Delta m_{1}+V(x) m_{1} \\
m_{1}(0, x) \equiv 1
\end{array}\right.
$$

(for the initial condition, see (1)). Here $\partial_{t}=\partial / \partial t$ is the time derivative, $\Delta$ is the discrete Laplacian acting on functions $\psi: \mathbb{Z}^{d} \rightarrow \mathbb{R}$ as

$$
\Delta \psi(x)=\frac{1}{2 d} \sum_{\left|x^{\prime}-x\right|=1} \psi\left(x^{\prime}\right)-\psi(x),
$$

and the function $V(x)$, called the random potential, is given by

$$
V(x):=V^{+}(x)-V^{-}(x), \quad x \in \mathbb{Z}^{d} .
$$

Remark. By the symmetry of the operator $H=\kappa \Delta+V(x)$ (which implies time reversibility of the system), the solution $m_{1}(t, x)$ of the problem (3) may be interpreted as the mean number of agents at site $x$ at time $t$, given that initially there is a uniform distribution of agents, $X(x) \equiv 1$ (i.e., one agent per site).

Similarly, for the local first-order moment $m_{1}=m_{1}(t, x, y)$ we obtain the same differential equation as in (3) but with a different (localized) initial condition:

$$
\left\{\begin{array}{l}
\partial_{t} m_{1}=\kappa \Delta m_{1}+V(x) m_{1} \\
m_{1}(0, x, y)=\delta_{y}(x)
\end{array}\right.
$$

Thus, the first-order quenched moments $m_{1}(t, x)$ and $m_{1}(t, x, y)$ are solutions to the Cauchy problems (3) and (5), respectively, which involves the celebrated Anderson operator $H=\kappa \Delta+V(x)$ (see Molchanov 1991, 1994). As explained in Gärtner \& 
Molchanov (1990), the intermittency properties of the solution are deeply related to the localization properties of the spectrum of the operator $H$. In our analysis of intermittency, we will not use this analogy.

Qualitatively speaking, the problem (3) describes the mean flow of agents in the presence of random sources (points where $V(x)>0$ ) and random sinks (points where $V(x)<0$ ). Two competing effects are present (cf. Section 1): the diffusion mechanism represented by the Laplace operator $\Delta$, and the branching mechanism represented by the random potential $V$. The diffusion tends to make the population density $m_{1}$ flat, while the potential makes it irregular by either inhibiting $(V<0)$ or enhancing $(V>0)$ the population growth.

\section{The Feynman-Kac formula}

In this section, we discuss the Feynman-Kac formula, which furnishes a useful probabilistic representation of the solutions to the Cauchy problems (3) and (5). For the sake of technical simplicity, from now on we assume that the pairs $V^{ \pm}(x)$ are independent and identically distributed (i.i.d.) for all $x \in \mathbb{Z}^{d}$, and in particular the potential $V(x)=V^{+}(x)-V^{-}(x)$ is also i.i.d. Then, under certain moment conditions on the potential $V$ (which are automatically satisfied for i.i.d. potentials with Weibull tails to be considered below), the solutions $m_{1}(t, x), m_{1}(t, x, y)$ admit the following Feynman-Kac representation (see Gärtner \& Molchanov 1990; Molchanov 1991):

$$
\begin{aligned}
m_{1}(t, x) & =\mathrm{E}_{x} \exp \left(\int_{0}^{t} V\left(x_{s}\right) \mathrm{d} s\right), \\
m_{1}(t, x, y) & =\mathrm{E}_{x} \exp \left(\int_{0}^{t} V\left(x_{s}\right) \mathrm{d} s\right) \delta_{y}\left(x_{t}\right) .
\end{aligned}
$$

Here $\mathrm{E}_{x}$ denotes expectation with respect to an auxiliary simple symmetric random walk $x_{t}$ started at $x$ (with the same jumping rate $\kappa$ ). Formulas (6) are in line with the "Lagrangian approach", where instead of looking at agents visiting a given point, one follows the motion of a tagged agent. The statistical weight $\exp \left(\int_{0}^{t} V\left(x_{s}\right) \mathrm{d} s\right)$ characterizes the "variation of mass" resulting from the interaction with the medium (Molchanov 1991).

Let us sketch the proof of formula $(6)$ for $m_{1}(t, x)$. Supposing that the function $m_{1}$ is given by (6), let us verify that it satisfies the differential equation (3) (obviously, (6) implies the initial condition in (3)). Using the Markov property ("lack of memory") 
and the local structure of transitions of the random walk $x_{t}$, we have

$$
\begin{aligned}
m_{1}(t & +h, x)=\mathrm{E}_{x} \exp \left(\int_{0}^{t+h} V\left(x_{s}\right) \mathrm{d} s\right) \\
& =\mathrm{E}_{x} \exp \left(\int_{0}^{h} V\left(x_{s}\right) \mathrm{d} s\right)\left[\mathrm{E}_{x_{h}} \exp \left(\int_{h}^{t+h} V\left(x_{s}\right) \mathrm{d} s\right)\right] \\
& =\mathrm{E}_{x} \exp \left(\int_{0}^{h} V\left(x_{s}\right) \mathrm{d} s\right) m_{1}\left(t, x_{h}\right) \\
& =\mathrm{E}_{x}\left(1+\int_{0}^{h} V\left(x_{s}\right) \mathrm{d} s+o(h)\right) m_{1}\left(t, x_{h}\right) \\
& =(1-\kappa h)(1+V(x) h+o(h)) m_{1}(t, x)+(1+O(h))\left(m_{1}\left(t, x_{h}\right)-m_{1}(t, x)\right) \\
& =m_{1}(t, x)+(V(x)-\kappa) h m_{1}(t, x)+\frac{\kappa h}{2 d} \sum_{\left|x^{\prime}-x\right|=1} m_{1}\left(t, x^{\prime}\right)+o(h) .
\end{aligned}
$$

Taking $m_{1}(t, x)$ to the left-hand side and dividing by $h \rightarrow 0$, we get (3).

\section{Lyapunov exponents for $m_{1}$}

From the Feynman-Kac representation (6), it is clear that the long-term behaviour of the solution is determined by large values of the potential $V(\cdot)$. Hence, the structure of the upper tail of the probability distribution of the potential is of principal importance. In this work, we shall consider the case of Weibull-type tails:

$$
\mathbb{P}\{V(x)>v\} \sim \exp \left(-c v^{\alpha}\right) \quad(v \rightarrow \infty),
$$

with some $\alpha>1, c>0$. For instance, normal distribution $\mathcal{N}\left(0, \sigma^{2}\right)$ belongs to this class, with $\alpha=2$.

Recall that the moment functions $m_{1}(t, x), m_{1}(t, x, y)$ depend on the random medium through the random potential $V(\cdot)$, and are therefore random variables. To investigate their asymptotic behaviour, let us consider the annealed statistical moments

$$
\left\langle m_{1}(t, x)^{p}\right\rangle, \quad\left\langle m_{1}(t, x, y)^{p}\right\rangle \quad(p=1,2, \ldots),
$$

where $\langle\cdot\rangle$ denotes expectation with respect to the environmental distribution $\mathbb{P}$.

The following result is essentially due to Gärtner \& Molchanov (1990) (see also Zeldovich et al. 1988 for an earlier version and Albeverio et al. 2000 for the part with $\left.m_{1}(t, x, y)\right)$.

Theorem 1. Assume that the random potential $V(\cdot)$ is i.i.d. and has a Weibull tail (7) with $\alpha>1$. Set $\alpha^{\prime}:=\frac{\alpha}{\alpha-1}>1$. Then the following limits (moment Lyapunov exponents) exist for both $m_{1}(t, x)$ and $m_{1}(t, x, y)$ :

$$
\lambda_{p}:=\lim _{t \rightarrow \infty} \frac{\log \left\langle m_{1}^{p}\right\rangle}{t^{\alpha^{\prime}}}=\gamma(\alpha, c) p^{\alpha^{\prime}} \quad(p=1,2, \ldots),
$$

where

$$
\gamma(\alpha, c)=(\alpha-1) \alpha^{-\alpha^{\prime}} c^{-1 /(\alpha-1)}
$$


Note that the right-hand side of equation (8) does not depend on the starting point $x$, which is obviously due to the medium's homogeneity. More striking is the fact that the Lyapunov exponents for the local moments $m_{1}(t, x, y)$ coincide with those for the total moments $m_{1}(t, x)$ (and thus are independent of the point $y$ as well). This shows that in the long run, all sites $y \in \mathbb{Z}^{d}$, no matter how far from the starting point $x$, make the same contribution to the growth of the annealed mean population size.

Let us sketch the proof of Theorem 1 for the total moment $m_{1}(t, x)$. Consider the cumulant generating function

$$
G(t):=\log \left\langle\mathrm{e}^{t V(x)}\right\rangle
$$

(since $V(\cdot)$ is i.i.d., the right-hand side does not depend on $x$ ). For a Weibull distribution (7) one can show that

$$
G(t) \sim \gamma(\alpha, c) t^{\alpha^{\prime}}, \quad t \rightarrow \infty
$$

where $\gamma(\alpha, c)$ is defined in (9). For example, if the distribution is given precisely by

$$
\mathbb{P}\{V(x)>v\}=\exp \left(-c v^{\alpha}\right) \quad(v \geq 0),
$$

then integration by parts gives

$$
\left\langle\mathrm{e}^{t V(x)}\right\rangle=\int_{0}^{\infty} \mathrm{e}^{t v} \mathrm{~d}\left(1-\mathrm{e}^{-c v^{\alpha}}\right)=-\int_{0}^{\infty} \mathrm{e}^{t v} \mathrm{~d}\left(\mathrm{e}^{-c v^{\alpha}}\right)=t \int_{0}^{\infty} \mathrm{e}^{t v-c v^{\alpha}} \mathrm{d} v
$$

By the Laplace asymptotic method, logarithmic asymptotics of the integral in (11) is determined by the maximum of the function $g(v)=t v-c v^{\alpha}$. Solving the equation

$$
g^{\prime}(v)=t-c \alpha v^{\alpha-1}=0
$$

yields that the maximum is attained at $v_{0}=(t / c \alpha)^{1 /(\alpha-1)}$. As a result,

$$
\log \left\langle\mathrm{e}^{t V(x)}\right\rangle \sim g\left(v_{0}\right)=t^{\alpha /(\alpha-1)} c^{-1 /(\alpha-1)}\left(\alpha^{-1 /(\alpha-1)}-\alpha^{-\alpha /(\alpha-1)}\right)=\gamma(\alpha, c) t^{\alpha^{\prime}},
$$

in accord with (10).

We are now able to derive a suitable lower bound. Let us keep in (6) only one path of the random walk $x_{s}$, namely the one that stays at its initial point $x$ up until time $t$. Since the sojourn time at $x$ exceeds $t$ with probability $\mathrm{e}^{-\kappa t}$, we get

$$
m_{1}(t, x) \geq \mathrm{e}^{-\kappa t} \cdot \mathrm{e}^{V(x) t}
$$

hence

$$
\left\langle m_{1}(t, x)^{p}\right\rangle \geq \mathrm{e}^{-p \kappa t}\left\langle\mathrm{e}^{p t V(x)}\right\rangle=\mathrm{e}^{-p \kappa t+G(p t)} .
$$

To obtain an upper bound asymptotically equivalent to the lower one, we apply 
Jensen's inequality and use Fubini's theorem as follows:

$$
\begin{aligned}
\left\langle m_{1}(t, x)^{p}\right\rangle & =\left\langle\left(\mathrm{E}_{x}\left[\exp \left\{\int_{0}^{t} V\left(x_{s}\right) \mathrm{d} s\right\}\right]\right)^{p}\right\rangle \\
& \leq\left\langle\mathrm{E}_{x}\left[\exp \left\{p \int_{0}^{t} V\left(x_{s}\right) \mathrm{d} s\right\}\right]\right\rangle \\
& \leq E_{x}\left\langle\frac{1}{t} \int_{0}^{t} \mathrm{e}^{p t V\left(x_{s}\right)} \mathrm{d} s\right\rangle \\
& =\frac{1}{t} \int_{0}^{t} \mathrm{E}_{x}\left\langle\mathrm{e}^{p t V\left(x_{s}\right)}\right\rangle \mathrm{d} s \\
& =\left\langle\mathrm{e}^{p t V(x)}\right\rangle=\mathrm{e}^{G(p t)} .
\end{aligned}
$$

The combination of the estimates (12) and (13) yields

$$
\frac{-\kappa t+G(p t)}{t^{\alpha^{\prime}}} \leq \frac{\log \left\langle m_{1}(t, x)^{p}\right\rangle}{t^{\alpha^{\prime}}} \leq \frac{G(p t)}{t^{\alpha^{\prime}}}
$$

and the result of Theorem 1 follows in view of (10).

In conclusion of this section, let us point out that Gärtner \& Molchanov (1990) have also evaluated the quenched Lyapunov exponent

$$
\tilde{\lambda}:=\lim _{t \rightarrow \infty} \frac{\log m_{1}}{t(\log t)^{1 / \alpha}}=\left(\frac{d}{c}\right)^{1 / \alpha}
$$

which exists $\mathbb{P}$-almost surely (i.e., with probability 1 ). Let us explain this result briefly, following Zeldovich et al. (1988). The crucial observation is that the main contribution to the expectation in (6) for large values of $t$ is provided by an optimal path of the random walk $x_{t}$, which travels at a distance of order of $t$ from the origin $x$ to reach the maximum value of the potential

$$
V_{0}=\max \{V(y):|y-x| \leq t\}
$$

(rather than by a typical path with $\left|x_{t}-x\right| \simeq t^{1 / 2}$ ). To estimate a typical value of the maximum $V_{0}$, note that the probability $p=\mathbb{P}\{V(y)>v\}$ to exceed a high level $v \rightarrow \infty$ at a given point $y$ equals $p=\mathbb{P}\{V(y)>v\} \simeq \exp \left(-c v^{\alpha}\right) \rightarrow 0$. Neglecting the unlikely possibility that the maximum value $V_{0}$ is attained at more than one point and using that there are about $N \simeq t^{d}$ integer points in the ball of radius $t$, from the relation $\mathrm{P}\left\{V_{0}>v\right\} \simeq N p \simeq 1$ we get the estimate

$$
V_{0} \simeq\left(\frac{d \log t}{c}\right)^{1 / \alpha} \quad(t \rightarrow \infty) .
$$

On the other hand, the probability of the optimal path can be shown to be asymptotically of order of $\exp \left(-c_{1} t\right)$. Inserting these estimates into formula (6), we obtain

$$
\log m_{1} \simeq-c_{1} t+t\left(\frac{d \log t}{c}\right)^{1 / \alpha} \sim t\left(\frac{d \log t}{c}\right)^{1 / \alpha} \quad(t \rightarrow \infty),
$$


and (14) follows.

Heuristically, the results (8) and (14) imply a super-exponential temporal growth as $t \rightarrow \infty$ :

$$
\left\langle m_{1}^{p}\right\rangle \approx \exp \left(\lambda_{1, p} t^{\alpha^{\prime}}\right), \quad m_{1} \approx \exp \left(\tilde{\lambda} t(\log t)^{1 / \alpha}\right)
$$

where " $\approx$ " denotes the logarithmic equivalence. Note that the time scale for the annealed moments $\left\langle m_{1}^{p}\right\rangle$ grows much faster than the time scale for the moment $m_{1}$ itself. This can be explained by the presence of extremely high peaks in the spatial distribution of the population density, which is an indicator of intermittency. In the next section, we will address the intermittency issue in more detail.

\section{Intermittency of $m_{1}$}

The main qualitative inference from Theorem 1 is that we deal here with a strongly intermittent structure. To see why, we note from (3) that the specific Lyapunov exponents $\lambda_{p} / p$ are given by

$$
\frac{\lambda_{p}}{p}=\gamma(\alpha, c) p^{\alpha^{\prime}-1}
$$

Note that by the Lyapunov inequality, for any $p<q$ we have

$$
\left\langle m_{1}^{p}\right\rangle^{1 / p} \leq\left\langle m_{1}^{q}\right\rangle^{1 / q}
$$

so that

$$
\frac{\log \left\langle m_{1}^{p}\right\rangle}{p} \leq \frac{\log \left\langle m_{1}^{q}\right\rangle}{q}
$$

This implies that the specific exponents $\lambda_{p} / p$ are always non-decreasing in $p$,

$$
\frac{\lambda_{p}}{p} \leq \frac{\lambda_{q}}{q} \quad(p<q)
$$

Moreover, from (16) it follows that $\lambda_{p} / p$ is strictly increasing as a function of $p$, since $\alpha^{\prime}>1$ :

$$
\lambda_{1}<\frac{\lambda_{2}}{2}<\frac{\lambda_{3}}{3}<\cdots
$$

which implies a progressive temporal growth of the moments:

$$
\left\langle m_{1}^{2}\right\rangle \gg\left\langle m_{1}\right\rangle^{2}, \quad\left\langle m_{1}^{3}\right\rangle \gg\left\langle m_{1}^{2}\right\rangle^{3 / 2}, \quad \text { etc. }
$$

As was first observed by Zeldovich et al. (1988) and argued more carefully by Gärtner \& Molchanov (1990), this is an indication of strong intermittency in the spatial distribution of $m_{1}$, and it is our aim to explain their argument here.

For definiteness, we shall speak of the total moment $m_{1}(t, x)$. Let us pick a number $r_{1}$ such that $\lambda_{1}<r_{1}<\lambda_{2} / 2$, and consider the (random) level sets on the lattice $\mathbb{Z}^{d}$ on which the magnitude of $m_{1}$ exceeds an exponentially high level:

$$
\Gamma_{r_{1}}(t):=\left\{x \in \mathbb{Z}^{d}: m_{1}(t, x)>\exp \left(r_{1} t^{\alpha^{\prime}}\right)\right\}
$$


The spatial density of points in the set $\Gamma_{r_{1}}(t)$ can be determined as the limit

$$
\rho_{r_{1}}(t):=\lim _{R \rightarrow \infty} \frac{\#\left(\Gamma_{r_{1}}(t) \cap B_{R}\right)}{\#\left(B_{R}\right)}
$$

where $B_{R}=\left\{x \in \mathbb{Z}^{d}:|x| \leq R\right\}$ is the "ball" of radius $R \rightarrow \infty$ and $\#(B)$ is the number of points $x \in \mathbb{Z}^{d}$ contained in the set $B \subset \mathbb{Z}^{d}$. By general results from ergodic theory (Birkhoff-Khinchin's theorem), the density $\rho_{r_{1}}(t)$ coincides with the probability that the set $\Gamma_{r_{1}}(t)$ contains a given site $x$ :

$$
\rho_{r_{1}}(t)=\mathbb{P}\left\{x \in \Gamma_{r_{1}}(t)\right\}=\mathbb{P}\left\{m_{1}(t, x)>\exp \left(r_{1} t^{\alpha^{\prime}}\right)\right\}
$$

Applying Chebyshev's inequality and using (15), we see that

$$
\begin{aligned}
\mathbb{P}\left\{m_{1}(t, x)>\exp \left(r_{1} t^{\alpha^{\prime}}\right)\right\} & \leq\left\langle m_{1}(t, x)\right\rangle \cdot \exp \left(-r_{1} t^{\alpha^{\prime}}\right) \\
& \approx \exp \left(\lambda_{1} t^{\alpha^{\prime}}\right) \cdot \exp \left(-r_{1} t^{\alpha^{\prime}}\right) \\
& =\exp \left(-\left(r_{1}-\lambda_{1}\right) t^{\alpha^{\prime}}\right) \rightarrow 0 \quad(t \rightarrow \infty)
\end{aligned}
$$

hence the spatial density of $\Gamma_{r_{1}}(t)$ is exponentially small. On the other hand, it is easy to see that the contribution to the second-order moment $\left\langle m_{1}(t, x)^{2}\right\rangle$ from points outside $\Gamma_{r_{1}}(t)$ is exponentially negligible:

$$
\begin{aligned}
\left\langle m_{1}(t, x)^{2} I\left\{x \notin \Gamma_{r_{1}}(t)\right\}\right\rangle & =\left\langle m_{1}(t, x)^{2} I\left\{m_{1}(t, x) \leq \exp \left(r_{1} t^{\alpha^{\prime}}\right)\right\}\right\rangle \\
& \leq \exp \left(2 r_{1} t^{\alpha^{\prime}}\right) \\
& \ll \exp \left(\lambda_{2} t^{\alpha^{\prime}}\right) \approx\left\langle m_{1}^{2}(t, x)\right\rangle
\end{aligned}
$$

(here $I\{A\}$ denotes the indicator of event $A$ ). That is to say, the second-order moment $\left\langle m_{1}^{2}\right\rangle$ is basically formed by high "overshoots" of $m_{1}(t, x)$ that occur on the set $\Gamma_{r_{1}}(t)$.

In a similar way, choosing $r_{2}$ such that

$$
\frac{\lambda_{2}}{2}<r_{2}<\frac{\lambda_{3}}{3}
$$

one can show that the third-order moment $m_{3}$ is essentially formed on the level set

$$
\Gamma_{r_{2}}(t)=\left\{x \in \mathbb{Z}^{d}: m_{1}(x, t)>\exp \left(\lambda_{2} t^{\alpha^{\prime}}\right)\right\} \subset \Gamma_{r_{1}}(t)
$$

and so on. Thus, there is a hierarchical sequence of spatially sparse level sets

$$
\Gamma_{r_{1}}(t) \supset \Gamma_{r_{2}}(t) \supset \cdots \supset \Gamma_{r_{p}}(t) \supset \cdots \quad\left(r_{1}<r_{2}<\cdots<r_{p}<\cdots\right)
$$

such that each moment $\left\langle m_{1}^{p}\right\rangle$ is generated by overshoots of the field $m_{1}(t, x)$ occurring on the respective set $\Gamma_{r_{p}}(t)$, which corresponds to the heuristic picture of intermittency. 


\section{Higher-order quenched moments}

In the previous sections we have seen that the moment approach proves quite efficient in the intermittency analysis of the first-order quenched moments $m_{1}(t, x)=\mathrm{E}_{x}^{\omega} X_{t}$, $m_{1}(t, x, y)=\mathrm{E}_{x}^{\omega} X_{t}(y)$, using their annealed moments $\left\langle m_{1}^{p}\right\rangle$.

The same method can be applied to studying the higher-order quenched moments

$$
m_{n}(t, x):=\mathrm{E}_{x}^{\omega} X_{t}^{n}, \quad m_{n}(t, x, y):=\mathrm{E}_{x}^{\omega} X_{t}(y)^{n}
$$

(see Albeverio et al. 2000). From the qualitative picture of intermittency explained above, it is natural to expect that the functions $m_{n}$ will appear strongly intermittent as well, the situation probably getting even more irregular as compared to the case of $m_{1}$. Our aim in this section is to present the results concerning $m_{n}$.

For the sake of clarity, let us assume that the annihilation rate $V^{-}$equals zero, so that the branching medium is completely determined by the random potential $V(\cdot)=$ $V^{+}(\cdot) \geq 0$. One can show (see Albeverio et al. 2000) that the functions $m_{n}$ satisfy the chain of evolution equations

$$
\left\{\begin{array}{l}
\partial_{t} m_{n}=\kappa \Delta m_{n}+V(x) m_{n}+V(x) f_{n}, \\
m_{n}(0, x) \equiv 1, \quad m_{n}(0, x, y)=\delta_{y}(x)
\end{array} \quad n=1,2, \ldots\right.
$$

where

$$
f_{n} \equiv f_{n}\left[m_{1}, \ldots, m_{n-1}\right]:=\sum_{i=1}^{n-1}\left(\begin{array}{c}
n \\
i
\end{array}\right) m_{i} m_{n-i}
$$

and $\left(\begin{array}{c}n \\ i\end{array}\right)=\frac{n !}{i !(n-i) !}$ are the binomial coefficients.

The initial-value problem (17) can be viewed as an inhomogeneous Cauchy problem for the Anderson operator $H=\kappa \Delta+V(x)$ (cf. (3), (5)). Similarly to the homogeneous case, the solution of this problem admits a representation of the Feynman-Kac type (see details in Albeverio et al. 2000); for example, $m_{n}(t, x)$ can be represented in the form (cf. (6))

$$
m_{n}(t, x)=m_{1}(t, x)+\mathrm{E}_{x} \int_{0}^{t} \exp \left(\int_{0}^{s} V\left(x_{u}\right) \mathrm{d} u\right) V\left(x_{s}\right) f_{n}\left(t-s, x_{s}\right) \mathrm{d} s .
$$

The higher-order annealed Lyapunov exponents, specifying the rate of super-exponential growth of $\left\langle m_{n}^{p}\right\rangle$, are given by the following theorem (Albeverio et al. 2000).

Theorem 2. Under the same assumptions as in Theorem 1 above (that is, i.i.d. $V(\cdot)$, Weibull tail (7) with $\alpha>1)$, for both $m_{n}(t, x)$ and $m_{n}(t, x, y)$ it holds

$$
\lambda_{n, p}:=\lim _{t \rightarrow \infty} \frac{\log \left\langle m_{n}^{p}\right\rangle}{t^{\alpha^{\prime}}}=\gamma(\alpha, c)(n p)^{\alpha^{\prime}} \quad(n=1,2, \ldots, p=1,2, \ldots)
$$

where $\alpha^{\prime}:=\frac{\alpha}{\alpha-1}>1$ and the constant $\gamma(\alpha, c)$ is the same as in Theorem $1($ see $(9))$. 
Note that for $n=1$, we have $\lambda_{1, p} \equiv \lambda_{p}$ (see Theorem 1), and formulas (18) are reduced to the expressions (8).

The quenched Lyapunov exponents of $m_{n}$ were evaluated by Molchanov (1996):

$$
\tilde{\lambda}_{n}:=\lim _{t \rightarrow \infty} \frac{\log m_{n}}{t(\log t)^{1 / \alpha}}=n\left(\frac{d}{c}\right)^{1 / \alpha} \quad(\mathbb{P} \text {-a.s. }),
$$

which is a generalization of formula (14).

Comparing the results (18) and (19), we note that the time scales for the annealed and quenched asymptotics of $m_{n}$ are quite different, the former growing much faster than the latter. This situation is analogous to that for the first-order moment $m_{1}$ (see Section 5), and again is an indication of intermittency. This is confirmed by progressive growth (in both $p$ and $n$ ) of the specific higher-order annealed Lyapunov exponents,

$$
\begin{aligned}
\frac{\lambda_{n, p}}{p} & =\gamma(\alpha, c) n^{\alpha^{\prime}} p^{\alpha^{\prime}-1} \\
\frac{\lambda_{n, p}}{n} & =\gamma(\alpha, c) n^{\alpha^{\prime}-1} p^{\alpha}
\end{aligned}
$$

\section{Adequacy of moments}

The principal qualitative conclusion from the previous analysis may seem somewhat disappointing, as it appears that the annealed moments are nonrepresentative (Molchanov 1991). Indeed, the moments of orders up to $p$ leave out information about higher peaks, which are only "visible" in the moments of orders $p^{\prime}>p$.

However, the mere comparison of the results (18) and (19) reveals the following remarkable relation between the various Lyapunov exponents responsible for the superexponential growth of the annealed moments:

$$
\lambda_{n, p}=\lambda_{1, n p} \quad(n=1,2, \ldots, p=1,2, \ldots) .
$$

Heuristically, the identity (20) implies that

$$
\left\langle m_{n}^{p}\right\rangle \approx\left\langle m_{1}^{n p}\right\rangle \quad(t \rightarrow \infty) .
$$

Thus, relative to the first-order moment $m_{1}$, the higher-order moments $m_{n}$ behave (almost) in a "regular" fashion. This observation makes it plausible that intermittency of $m_{n}$ (and possibly of the underlying population density $X_{t}$ as well) essentially amounts to that of the first-order moment $m_{1}$ (cf. Molchanov 1996, where a similar observation was made).

This conjecture is further confirmed by the comparison of the quenched Lyapunov exponents (14) and (19):

$$
\tilde{\lambda}_{n}=n \tilde{\lambda}_{1} \quad(\mathbb{P} \text {-a.s. }),
$$

so that, with probability 1 ,

$$
m_{n} \approx m_{1}^{n} \quad(t \rightarrow \infty)
$$


Note that the overall (annealed) probability measure $\mathcal{P}_{x}$, defining the population process $X_{t}$ in random branching medium $V^{ \pm}$, can be symbolically represented by the total probability formula:

$$
\mathcal{P}_{x}(\cdot)=\int_{\{\omega\}} \mathrm{P}_{x}^{\omega}(\cdot) \mathbb{P}(\mathrm{d} \omega)=\left\langle\mathrm{P}_{x}^{\omega}(\cdot)\right\rangle .
$$

We conjecture that the following is true:

Adequacy Principle. The ratio

$$
\rho(t):=\frac{X_{t}}{m_{1}(t, x)}
$$

is bounded in $\mathcal{P}$-probability from above and below, uniformly in $t \geq 0$, i.e., for any $\varepsilon>0$ there exists $\delta>0$ such that for all $t \geq 0$

$$
\mathcal{P}\left\{\delta \leq \rho(t) \leq \delta^{-1}\right\} \geq 1-\varepsilon .
$$

Note that from the Adequacy Principle it would follow that

$$
\frac{\log X_{t}}{\log m_{1}(t, x)} \stackrel{\mathcal{P}}{\longrightarrow} 1 \quad(t \rightarrow \infty),
$$

provided that

$$
m_{1}(t, x) \stackrel{\mathbb{P}}{\longrightarrow} \infty \quad(t \rightarrow \infty) .
$$

In turn, this would imply the equality of the (quenched) Lyapunov exponents for $X_{t}$ and $m_{1}(t, x)$ : if for some deterministic scale function $A(t) \uparrow \infty$ one has

$$
\lim _{t \rightarrow \infty} \frac{\log m_{1}(t, x)}{A(t)}=1 \quad(\mathbb{P} \text {-a.s. }),
$$

then it follows that

$$
\lim _{t \rightarrow \infty} \frac{\log X_{t}}{A(t)}=1 \quad(\mathcal{P} \text {-a.s. }) .
$$

We will address these issues elsewhere.

\section{Concluding remarks}

In this paper, we have focused on the notion of intermittency, referring to the formation of irregular spatio-temporal structures in complex systems, with the view of potential applications in urban and regional modelling. We have demonstrated that the model of branching random walk with random branching rates may produce a strongly pronounced intermittency of the (quenched) mean population distribution $m_{1}$ in space and time. In so doing, progressive growth of annealed Lyapunov exponents $\lambda_{1, p}$, with respect to the moment order $p$, proves to be a simple yet efficient test of intermittency. Let us emphasize that, due to randomness of the medium, intermittency arises here in a linear problem of the form $\partial_{t} u=H u$, although the actual mechanism of intermittency 
is a nonlinear dependence of the solution, through the Feynman-Kac representation, on the random coefficients of the equation.

We have also considered the higher-order quenched moments $m_{n}$, for which the similar technique is available. In particular, the higher-order Lyapunov exponents $\lambda_{n, p}$ may be evaluated, confirming the intermittent nature of the process. A remarkable identity between the various Lyapunov exponents, $\lambda_{n, p}=\lambda_{1, n p}$, suggests that the annealed moments $\left\langle m_{n}^{p}\right\rangle$ grow in a "regular" fashion relative to the first-order moment. Therefore, although intermittent structures are very hard to deal with statistically, the main work to be done relates to the first-order moment only, which seems to be a good news (relative to the "bad" news of intermittency!).

Needless to say, the model we have discussed is far too idealized and simplified to be of any practical use. There are also further mathematical issues to be addressed in order to gain a better understanding of intermittency, including its fine spatial structure. In more realistic models, proper modifications should be made to allow for other types of probability distribution of the potential, and also for finiteness of space and time, spatial capacity constraints, temporal variability and spatial correlations of the medium, and interaction between the agents.

Some of these issues are still open, while some other have already been addressed in the literature, for example:

- continuous-space models (see Gärtner \& König 2000);

- dependence of the spatial structure of peaks on the distribution tail of the potential (see Gärtner \& Molchanov 1998; Biskup \& König 2001);

- time-dependent models with short temporal correlations (see Carmona \& Molchanov 1994);

- spatial correlation structure of the quenched moment (see Gärtner \& den Hollander 1999).

However, our principal aim here has been to provide a quick introduction to intermittency as part of random media theory, in order to show that random media models may be of great relevance and value when it comes to understanding and explanation of various anomalous effects. The main qualitative message from our analysis to applied scientists and practitioners, and in particular to urban modellers, is that in an intermittent situation the usual statistical moments may not be representative of the actual process under consideration, so greater caution is needed when using the standard sampling techniques (based on averaging etc.). One practical advice based on the moment test is that should the higher-order moments, as functions of time, appear to be growing progressively in their order (which may be manifested, for instance, through "instability" or "divergence" of statistical and numerical estimates), then this may be a precursor of intermittency.

\section{Acknowledgements}

Partial support from the organizers of the International Conference on the Dynamics of Complex Urban Systems (Ascona, Centro Monte Verità, 4-6 November 2004), where 
this paper was presented, is gratefully acknowledged. I am pleased to express my deep gratitude to Stanislav Molchanov for numerous illuminating conversations over the years on many issues in random media theory and to Sergio Albeverio for stimulating collaboration. I have also appreciated interesting discussions on various aspects in urban applications with Peter Allen, Mike Batty, Itzhak Benenson, Mark Birkin, Volker Jentsch, Juval Portugali, and Damian Zanette. Special thanks are due to Sir Alan Wilson for his seminal books promoting good use of mathematics in urban and regional modelling.

\section{References}

[1] Albeverio, S., Bogachev, L.V., Molchanov, S.A., and Yarovaya, E.B. (2000). Annealed moment Lyapunov exponents for a branching random walk in a homogeneous random branching environment. Markov Process. Relat. Fields 6, 473-516.

[2] Allen, P.M., Deneubourg, J.L., Sanglier, M., Boon, F., and de Palma, A. (1978). The dynamics of urban evolution. 1: Interurban evolution; 2: Intraurban evolution. Final Report to the US Department of Transportation. Washington, D.C.

[3] Allen, P.M. and Sanglier, M. (1979). A dynamic model of growth in a central place system. Geograph. Anal. 11, 256-272.

[4] Biskup, M. and König, W. (2001). Long-time tails in the parabolic Anderson model with bounded potential. Ann. Probab. 29, 636-682.

[5] Bogachev, L.V. (2006). Random walks in random environments. In Encyclopedia of Mathematical Physics (Françoise, J.P., Naber, G., and Tsou, S.T., eds) 4, 353371. Elsevier, Oxford.

[6] Bouchaud, J.-P. and Georges, A. (1990). Anomalous diffusion in disordered media: Statistical mechanisms, models and physical applications. Phys. Rep. 195, no. 4-5, $127-293$.

[7] Bracken, A.J. and Tuckwell, H.C. (1992). Simple mathematical models for urban growth. Proc. Roy. Soc. London Ser. A 438, 171-181.

[8] Carmona, R.A. and Molchanov, S.A. (1994). Parabolic Anderson Problem and Intermittency. (Memoirs of the American Mathematical Society; No. 518.) American Math. Society, Providence, RI.

[9] Gärtner, J. and den Hollander, F. (1999). Correlation structure of intermittency in the parabolic Anderson problem. Probab. Theory Relat. Fields 114, 1-54.

[10] Gärtner, J. and König, W. (2000). Moment asymptotics for the continuous parabolic Anderson model. Ann. Appl. Probab. 10, 192-217.

[11] Gärtner, J. and Molchanov, S.A. (1990). Parabolic problems for the Anderson model. I: Intermittency and related topics. Comm. Math. Phys. 132, 613-655.

[12] Gärtner, J. and Molchanov, S.A. (1998). Parabolic problems for the Anderson model. II: Second-order asymptotics and structure of high peaks. Probab. Theory Relat. Fields 111, 17-55. 
[13] Giardina, I. and Bouchaud, J.-P. (2003). Bubbles, crashes and intermittency in agent based market models. Eur. Phys. J. B, Condens. Matter Phys. 31, 421-437.

[14] Gihman, I.I. and Skorohod, A.V. (1975). The Theory of Stochastic Processes 2. Springer, Berlin.

[15] Haken, H. (1978). Synergetics: An Introduction. Springer, Berlin.

[16] Kozlov, S.M. (1985). The method of averaging and walks in inhomogeneous environments. Russian Math. Surveys 40, no. 2, 73-145.

[17] Manrubia, S.C. and Zanette, D.H. (1998). Intermittency model for urban development. Phys. Rev. E 58, 295-302.

[18] Mikhailov, A.S. and Loskutov, A.Yu. (1991). Foundations of Synergetics II: Complex Patterns. Springer, Heidelberg.

[19] Molchanov, S.A. (1991). Ideas in the theory of random media. Acta Appl. Math. 22, 139-282.

[20] Molchanov, S.A. (1994). Lectures on random media. In: D. Bakry, R.D. Gill, and S.A. Molchanov. Lectures on Probability Theory. Ecole d'Eté de Probabilités de Saint-Flour XXII-1992 (Bernard, P., ed.). Lecture Notes Math. 1581, 242-411. Springer, Berlin.

[21] Molchanov, S.A. (1996). Reaction-diffusion equations in the random media: Localization and intermittency. In Nonlinear Stochastic PDEs. Hydrodynamic Limit and Burgers' Turbulence (Funaki, T. and Woyczynski, W.A., eds), 81-109. Springer, Berlin.

[22] Monin, A.S. and Yaglom, A.M. (1975). Statistical Fluid Mechanics: Mechanics of Turbulence 2. MIT, Cambridge, MA.

[23] Nicolis, G. and Prigogine, I. (1977). Self-Organization in Nonequilibrium Systems: From Dissipative Structures to Order through Fluctuations. Wiley, New York.

[24] Nicolis, G. and Prigogine, I. (1989). Exploring Complexity: An Introduction. W. H. Freeman, New York.

[25] Portugali, J. (2000). Self-Organization and the City. Springer, Berlin.

[26] Wilson, A.G. (1981). Catastrophe Theory and Bifurcation: Applications to Urban and Regional Systems. Groom Helm, London.

[27] Wilson, A.G. (2000). Complex Spatial Systems: The Modern Foundations of Urban and Regional Analysis. Pearson Education, Harlow.

[28] Zanette, D.H. and Manrubia, S.C. (1997). Role of intermittency in urban development: A model of large-scale city formation. Phys. Rev. Lett. 79, 523-526.

[29] Zanette, D.H. and Manrubia, S.C. (1998). Reply to: Comment on "Role of intermittency in urban development: A model of large-scale city formation". Phys. Rev. Lett. 80, 4831.

[30] Zeldovich, Ya.B., Molchanov, S.A., Ruzmaikin, A.A., and Sokoloff, D.D. (1988). Intermittency, diffusion and generation in a nonstationary random medium. Sov. Sci. Rev., Sect. C: Math. Phys. Rev. 7, 1-110. 